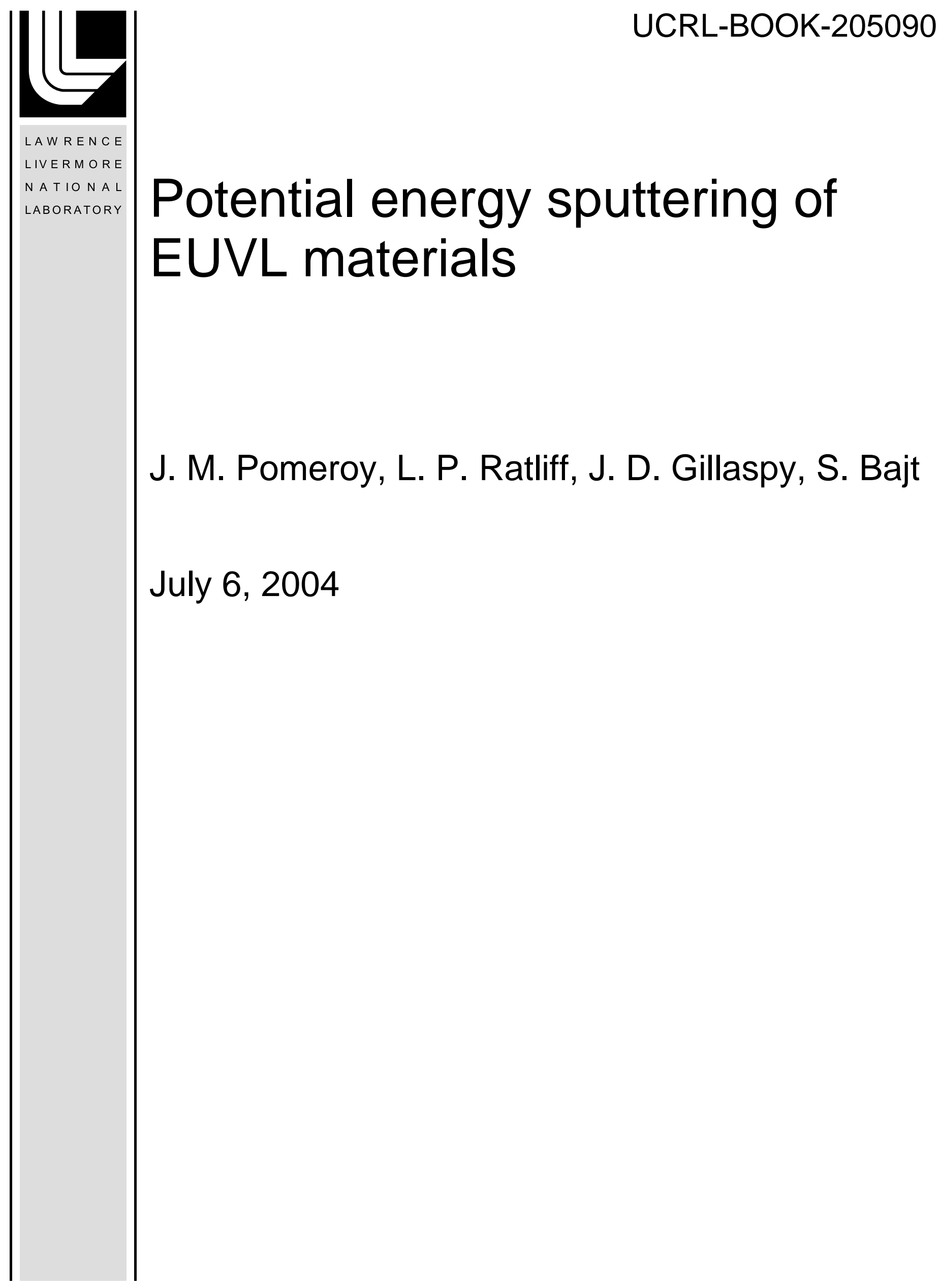


This document was prepared as an account of work sponsored by an agency of the United States Government. Neither the United States Government nor the University of California nor any of their employees, makes any warranty, express or implied, or assumes any legal liability or responsibility for the accuracy, completeness, or usefulness of any information, apparatus, product, or process disclosed, or represents that its use would not infringe privately owned rights. Reference herein to any specific commercial product, process, or service by trade name, trademark, manufacturer, or otherwise, does not necessarily constitute or imply its endorsement, recommendation, or favoring by the United States Government or the University of California. The views and opinions of authors expressed herein do not necessarily state or reflect those of the United States Government or the University of California, and shall not be used for advertising or product endorsement purposes.

This work was performed under the auspices of the U.S. Department of Energy by University of California, Lawrence Livermore National Laboratory under Contract W-7405-Eng-48. 


\title{
7.11 Potential energy sputtering of EUVL materials
}

\author{
Joshua M. Pomeroy, L.P. Ratliff, and J.D. Gillaspy \\ National Institute of Standards and Technology \\ Gaithersburg, MD 20899-8421
}

S. Bajt

Lawrence Livermore National Laboratory

Livermore, CA 94550

\section{Outline}

\subsubsection{Introduction}

7.11.2 Interactions of Highly Charged Ions with Solids

7.11.3 Experimental Studies of Potential Energy Damage to EUVL devices

7.11.4 Implications and Outlook

7.11.5 Summary

\subsubsection{Introduction}

Of the many candidates employed for understanding the erosion of critical Extreme Ultraviolet Lithography (EUVL) components, potential energy damage remains relatively uninvestigated. Unlike the familiar kinetic energy sputtering, which is a consequence of the momentum transferred by an ion to atoms in the target, potential energy sputtering occurs when an ion rapidly collects charge from the target as it neutralizes. Since the neutralization energy of a singly charged ion is typically on the order of $10 \mathrm{eV}$, potential energy effects are generally neglected for low charge state ions, and hence the bulk of the sputtering literature. As an ion's charge state is increased, the potential energy (PE) increases rapidly, e.g. $\mathrm{PE}\left(\mathrm{Xe}^{1+}\right)=11 \mathrm{eV}, \mathrm{PE}\left(\mathrm{Xe}^{10+}\right)=810 \mathrm{eV}, \mathrm{PE}\left(\mathrm{Xe}^{20+}\right)=4.6 \mathrm{keV}$, etc. ${ }^{1}$ By comparison, the binding energy of a single atom on a surface is typically about $5 \mathrm{eV}$, so even relatively inefficient energy transfer mechanisms can lead to large quantities of material being removed, e.g. $25 \%$ efficiency for $\mathrm{Xe}^{10+}$ corresponds to $\sim 40$ atoms/ion. By comparison, singly charged xenon ions with $\sim 20 \mathrm{keV}$ of kinetic energy sputter only about 5 atoms/ion at normal incidence, and less than 1 atom/ion at typical EUV source energies.

EUV light sources are optimized for producing approximately $10^{16}$ xenon ions per shot with an average charge state of $q=10$ in the core plasma ${ }^{2}$. At operational rates of $\sim 10$ $\mathrm{kHz}$, the number of ions produced per second becomes a whopping $10^{20}$ Even if only one in a billon ions reaches the collector, erosion rates could reach $\sim 10^{12}$ atoms per second, severely reducing the collector lifetime (for an average yield of 10 atoms/ion). In addition, efforts to reduce contamination effects may contribute to reduced neutralization and even larger potential energy damages rates (discussed further below). In order to provide accurate estimates for collector lifetimes and to develop mitigation schemes, NIST is working to understand and quantify potential energy damage mechanisms on materials relevant to EUVL. Accurate potential energy damage rates can then be used for 
projecting component lifetimes as source plasma conditions are modified and characterized.

This chapter will serve to provide an introduction and some background to the physics of highly charged ions and some of the relevant experimental work in the literature. This chapter will first provide a brief background and an overview of the interaction of highly charged ions (HCIs) with solids as it is currently understood. Secondly, it will present current data from screen test measurements performed to isolate and evaluate the effects of potential energy damage on critical EUVL materials. We will then speculate on the implications of work to date and the outlook for EUVL development and, finally, summarize.

\subsubsection{Interactions of Highly Charged Ions with Solids}

When singly charged ions interact with solids, the transfer of the ions' forward momentum is the dominant damage-forming mechanism, creating lattice dislocation and sputtered atoms. Kinetic energy sputtering is a thoroughly studied and well understood process for most elements and kinetic energies. Extensive experimental work has generated data on sputter rates as a function of kinetic energy for nearly every known combination of elements in the periodic table ${ }^{3}$. The compilation of these data resulted in the accumulation of accurate parameters for use in analytical fits like Yamamura's semi empirical model (based on Sigmund's theory of sputtering ${ }^{4}$ ). With increased interest in technologies that employ ion energies nearer to sputtering thresholds and at non-normal incidences, the models have been refined to increase their accuracy in the low energy and light ion regimes ${ }^{5}$. In addition to semi-empirical fits to actual data, the Monte Carlo simulation SRIM (TRIM) has been widely tested and accepted as an accurate benchmark for quantitatively describing ion-solid interactions, particularly stopping ranges and sputter yields ${ }^{6}$. In recent history, SRIM has been used to generate accurate predictions at arbitrary energies and incidence conditions for even further refinement of semi-empirical formulae that more accurately model the low energy (threshold) regime and the low mass ratio regime ${ }^{7}$. This vast compilation of knowledge, fit functions, and simulations make estimation of kinetic energy damage relatively easy and accurate, but they neglect charge and potential energy effects entirely.

The effects of kinetic energy, which are the leading order effects for singly charged ions, are still present during interactions of HCIs with surfaces, but are not necessarily the most significant effect. As many electrons are removed from an atom, the charge imbalance leads to enormous electric fields, e.g. the $1 \mathrm{~s}$ electron on $\mathrm{A}^{17+}$ ion will feel a $\sim 5 \times 10^{13} \mathrm{~V} / \mathrm{cm}$ electric field. The ion's enormous electric field interacts with the surface from many Bohr radii away, tearing electrons from the surface well before the ion interacts kinematically with the surface ${ }^{8}$. These extracted electrons are captured by the ion into atomic energy levels similar to the energy level the electron occupied in the solid, e.g. similar to the work function. These atomic energy levels tend to be very highlying Rydberg states ${ }^{9}$, e.g. $\mathrm{Xe}^{25+}$ on Au would capture electrons into levels where $\mathrm{n}>20$. Electrons captured into highly excited states with potentially high angular momentum numbers will relax by cascading to lower $\mathrm{n}$ levels, but the transition rates are highest 
when momentum is conserved by ejecting another electron. For example, in the process of one electron moving from the $n=20$ down to an $n=15$ level, 5 other electrons may be ejected from the ion, which will subsequently be replaced by five more electrons from the surface. Measurements of secondary electron yields from HCI-surface interactions have found that a HCI can "pump" hundreds of electrons per ion, many times the HCI's initial charge state ${ }^{10}$. Electronic extraction of this magnitude corresponds to enormous macroscopic analogs, for example, if a $\mathrm{Xe}^{20+}$ ion requires $\sim 100$ electrons from a surface to become completely neutralized, and the entire charge transfer occurs in an area $1 \mathrm{~nm}^{2}$ and $\sim 0.1 \mathrm{ps}^{*}$, this corresponds to a current density of $\sim 1 \times 10^{10} \mathrm{~A} / \mathrm{cm}^{2}$. With current densities of this magnitude, it is easy to see how the charge transfer is one way an HCI can de-stabilize a surface (depending on material, bulk vaporization will occur between $10^{5} \mathrm{~A} / \mathrm{cm}^{2}$ and $\left.10^{8} \mathrm{~A} / \mathrm{cm}^{2}\right)^{11}$.

The process of neutralizing highly charged ions on surfaces is known to dramatically destabilize some surfaces, as has been demonstrated by large secondary electron measurements, extremely high sputter yields (in diverse classes of materials, ranging from $\mathrm{SiO}_{2}, \mathrm{LiF}, \mathrm{GaAs}$, and $\left.\mathrm{UO}_{2}\right)^{8}$, similarly large secondary ion yields ${ }^{12}$, and $\mathrm{X}$-ray emission measurements from target materials during HCI exposure ${ }^{13}$. A unified theory that explains all these results has not yet been presented, but a few theories that have captured significant attention are worth summarizing.

Perhaps the most intuitive model for target damage due to HCIs simply suggests that the rapid charge transfer from the surface can locally deplete electrons in the solid. The residual positive charge expands due to the repulsive Coulomb forces resulting in a potentially massive explosion that removes many more atoms than the initial charge state $^{14}$. This "Coulomb explosion" model has persisted in part because of its ability to explain the large number of neutral atoms removed due to the HCI's neutralization, as was shown in a molecular dynamics simulation where charge was pinned on a predetermined geometry of surface atoms ${ }^{15}$. While the principal weakness of the Coulomb explosion model is its reliance on hole lifetimes long enough to develop a shockwave ${ }^{16}$, the model captures the principal idea that the HCI, through some mechanism, can introduce a shock into the target that results in significantly elevated damage rates by comparison to only kinetic energy effects.

Depending on the target material of interest, mechanisms other than Coulomb repulsion can be identified that could generate a shock wave resulting in massive surface damage. In covalent solids like III-V materials, $\mathrm{Si}$ and $\mathrm{SiO}_{2}, \mathrm{HCIs}$ can induce a structural instability leading to a shock wave electronically by "ultra-fast electronic excitation ${ }^{17}$." These materials are stable solids due to the binding nature of the valence bands, but the conduction bands are strongly anti-bonding. If enough carriers (approximately 1 per surface atom in the region of interest) are promoted from the valence to the conduction band, the equilibrium lattice spacing can grow significantly, introducing a severe internal

\footnotetext{
* The exact neutralization time will depend on experimental conditions and the properties of the neutralizing surface. This estimate is based on a relatively slow ion's drift time from the distance of first electron capture (classic over-the-barrier method) ${ }^{9}$ to penetration into the solid.
} 
stress $^{18}$. This stress can provide enough internal energy to significantly increase sputter yields, which may explain the large yields seen in materials like $\mathrm{GaAs}^{19}$.

The most dramatic potential sputtering effects have been reported in the alkali halides, particularly LiF, where sputter yields increased by a factor of $~ 30$ when the charge state was increased from $\mathrm{Ar}^{1+}$ to $\mathrm{Ar}^{11+}$ at $1 \mathrm{keV}$ of kinetic energy, with total sputter yields exceeding 80,000 amu/ion for $\mathrm{Xe}^{27+}$ at $1 \mathrm{keV}$ of kinetic energy ${ }^{20,21}$. A "defect-mediated desorption" model (DMD) has been employed to explain these data ${ }^{21}$, leveraging earlier work indicating that self-trapped excitons (STEs) in these systems could decay into lattice defects ${ }^{22}$. If the HCI's intense electronic interaction with the $\mathrm{LiF}$ target produces high densities of electron-hole pairs, these can decay into sub-surface $\mathrm{F}_{2}$ molecules and STEs. Each of these can then decay such that a fluorine atom escapes into the vacuum, and a neutral lithium atom remains on the surface. Subsequent ion-surface interactions stimulate desorption of the lithium preventing the growth of a segregated overlayer.

The defect mediated desorption model provides a plausible explanation for materials with strong electron-phonon coupling, but further extension is required to incorporate dramatic potential energy effects observed in materials like $\mathrm{MgO}$, which do not have strong electron-phonon coupling. The principal proponents of DMD argue that the lattice defects created by the kinetic energy of the incident ion allow electronic energy to become localized in the target, thereby providing a mechanism for large yields due to potential energy ${ }^{23}$. The proponents argue that this explains the apparent absence of potential sputtering in the limit of zero kinetic energy and the strong dependence of the sputtering yields on both kinetic energy and charge state.

While each of the potential sputtering models summarized here has directly dealt with only the potential energy, much of the experimental data suggest a synergy of kinetic and potential energies ${ }^{24}$. While the ion's interaction time with the surface prior to collision is determined by the kinetic energy and charge, it seems that the deposition of potential energy into the surface prior to the collision cascade pre-softens the surface, resulting in much higher yields of ejected material, i.e. potential energy transfer enhances kinetic energy sputtering. It has also been suggested that the collision process may produce lattice defects that allow electronic energy to be localized (STEs) resulting in an enhanced DMD process ${ }^{25}$, i.e. kinetic energy enhances potential sputtering.

It is clear that at higher charge states, the interaction of the ion with target materials becomes more intense and complicated, involving many more mechanisms at nonnegligible rates. Of all the materials classes studied, the noble metals are the only materials that have not shown clear and convincing evidence of potential energy related damage, but potential energy effects are not conclusively excluded either. Essentially all low conductivity materials studied have shown some sort of susceptibility to potential energy damage, including metal oxides ${ }^{26}$ and semi-metals ${ }^{27}$.

Selection of appropriate materials for use in a plasma environment requires balancing the relative significance of many different mechanisms, e.g. materials least susceptible to potential energy damage may be most susceptible to kinetic energy damage, attempts to 
screen critical components with gas curtains, etc. may actually increase component oxidation rates and therefore increase potential energy damage rates. Further studies on actual devices to quantify relative rates of damage are critical for accurate projection of component lifetimes and development of schemes for mitigating expected damage.

\subsubsection{Experimental Studies of Potential Energy Damage to EUVL devices}

The Electron Beam Ion Trap (EBIT) at the National Institute of Standards and Technology (NIST) is capable of producing very highly charged ions (e.g. $\mathrm{Bi}^{73+}$ ) and delivering monoenergetic beams of a particular charge state onto target samples. Highly charged ions can be created from a wide range of elements, over a broad range of charge states and delivered onto samples via a complex ultra-high vacuum ion beam line (described in detail elsewhere ${ }^{28,29}$ ). The NIST EBIT is being used in support of EUVL to isolate potential energy damage effects by exposing candidate materials and actual EUVL optics $^{30}$ to controlled doses of highly charged ions (HCIs) and then characterizing the effects with in situ scanning tunneling microscopy (STM) and ex situ EUV reflectometry.

An example of an EUV reflectance map is shown in Figure 1 of a prototype piece of EUVL optic after exposure to a very low dose of $\mathrm{Xe}^{44+}\left(\sim 1\right.$ ion per $\left.250 \mathrm{~nm}^{2}\right)$. The reflectivity of the optic is changed by $\sim 0.8 \%$ in the lower part of the figure, corresponding to the region where the optic was exposed to the xenon ions. A similar optic exposed to $\sim \mathrm{Xe}^{10+}$ ion per $10 \mathrm{~nm}^{2}$ showed a $0.3 \%$ change in the EUV reflectivity (not shown). Data of this type suggest that the reflectivity of an EUV optic is initially changed $1 \%$ for every $\sim 250 \mathrm{eV} / \mathrm{nm}^{2}$ of potential energy delivered to the optical surface ${ }^{\dagger}$. Using the bulk densities for the ruthenium oxides, this implies that $\sim 20 \mathrm{eV}$ of potential energy is required for the removal of each molecule. Using an estimated bulk binding energy of $\sim 5 \mathrm{eV}$, we can infer that $\sim 25 \%$ of an ion's potential energy is converted to sputtered material. Efficiencies of this magnitude are similar to computational results of damage due to potential energy effects ${ }^{15}$.

The reflectivity data is taken on samples that are part of a series of EUV optics exposed to the same number of HCIs per unit area and studied by STM and tunneling spectroscopy. For this series, the optics were exposed to $\sim 5 \times 10^{9} \mathrm{~mm}^{-2}$, or 1 ion per $\sim 200$ $\mathrm{nm}^{2}$. A $200 \mathrm{~nm}$ x $200 \mathrm{~nm}$ sample image of the EUV optic's surface after exposure to $\mathrm{Xe}^{10+}$ ions is shown at left in Figure 2. Extensive analysis of the surface topography does not reveal any characteristic feature(s) that can be correlated with an individual ion's impact. This can be understood after comparing the time scale of the ion exposure with the time scale for intrinsic surface smoothing. The ion exposure took place over the course of an hour, and the subsequent imaging in the STM takes place over several hours. While this is not long enough for the surface to react with any contaminants present in the

\footnotetext{
${ }^{\dagger}$ For these measurements, the EUV reflectivity initially increases as the capping layer, which inhibits reflectivity, is reduced. Continued erosion would result in a maximum of reflectivity and a subsequent reduction to values much less than the initial reflectivity. This non-functional (double-valued) dependence introduces an ambiguity in the analysis; the solution of least damage is assumed, actual damage may be $\sim 2$ times worse.
} 
ultra-high vacuum environment, the surface kinetics can wash out any characteristic feature, so the signature is lost in the intrinsic roughness.

While individual topographic features due to HCI impacts are not evident in the topographic images, spatially localized spectral features in the surface conductivity maps at moderate bias $(-0.63 \mathrm{~V})$ are consistent in density with the ion dose and consistent in size with analysis of the EUV reflectivity data. A representative $20 \mathrm{~nm} \times 20 \mathrm{~nm}$ spectral map is shown at right in Figure 2. This image represents the current at $-0.63 \mathrm{~V}$ of bias as a function of position, since the bias is negative, bright areas represent poor conduction and vice-versa. The dark cross-shaped features ${ }^{*}$ are patches of the surface with significantly higher conductivity than the surrounding region that may be due to single ion impacts. These individual features collectively represent a measurable change in the surface that we have statically analyzed.

While we have not seen quantifiable potential energy damage in the surface topography on the EUV optics, statistical analysis of the tunneling spectroscopy reveals an increase in the surface conductivity with increasing potential energy, shown in Figure 3. The optic's surface conductivity systematically increases with the potential energy deposited per ion ${ }^{\S}$. This is likely due to potential energy ablation of oxide on the surface. The higher potential energy density corresponds to a larger fraction of the surface's oxide being removed. Since the oxide is not deliberately grown, but is a consequence of exposure to atmosphere, the surface is rough on the nanometer scale prior to HCI exposure (nominally the same as Figure 2, left side). This roughness has a $\sim 5 \mathrm{~nm}$ characteristic lateral feature size, which may be indicative of oxide clusters (root mean square roughness $<1 \mathrm{~nm}$ ). An incident HCI may remove some oxide clusters whole, particularly if they are weakly bonded to the underlying capping layer ${ }^{31}$. This cluster dissociation idea is one possible model for the removal of large amounts of material per incident ion.

The HCI dose used in this study (Figure 3) was selected to avoid saturation effects at high charge states, higher doses of low charge states are expected to lead to damage which scales with the potential energy. We do not believe that the suggestion of any threshold is an accurate interpretation of the data. Initial data from much higher doses of $\mathrm{Xe}^{10+}$ on EUV optics suggest that damage scales with total potential energy dose, rather than strictly with charge state (or potential energy per ion).

This work indicates that the EUV optic materials are susceptible to potential sputtering as they are currently deployed. Whether optic lifetimes will ultimately be limited by effects of potential energy erosion will largely be determined by the source's operational characteristics. Since EUV sources do not yet meet the industrially required power output, demanding dramatic changes in design and operation, proper consideration of

\footnotetext{
$\ddagger$ The cross-like shape is due to nearest-neighbor filtering used to reduce pixel noise

$\S$ The error bars represent the propagated RMS values of the distribution of conductivities for mean value conductivity analysis. We are currently pursuing more advanced analysis that is expected to be more sensitive to the conductivity change, thereby reducing the relative errors.
} 
potential energy and the associated damage when minimizing risk to critical components is prudent.

\subsubsection{Implications and Outlook}

Evidence of HCI damage on EUV optics taken in concert with data listed in the references presented in this paper clearly indicates the existence of potential energy damage effects. EUV devices, as currently optimized for use in production tools, are susceptible to potential energy damage. It is possible to minimize or mitigate damage to the components by seeking to "harden" the components, or by minimizing the flux of HCIs from plasma sources so that potential energy damage is negligible compared with the kinetic energy effects. In either case, accurate quantitative data for damage rates of various materials is highly desirable, since accurate modeling of component lifetimes rely on accurate rate estimates.

Efforts to determine if sources currently in testing are emitting substantial fluxes of highly charged ions, or ions with substantial energies, are not conclusive. Some source suppliers indicate ion emission with kinetic energies as high as $20 \mathrm{keV}$, while others are adamant their source is not emitting ions above $500 \mathrm{eV}$. As sources are ramped up to production power levels and repetition rates, substantial ion emission may become more likely. Whether or not potential energy damage will be a limiting factor in production tools will ultimately depend on the source's ion emission rates, but prudent assessment of materials properties can allow for last minute corrections that might mitigate a showstopping oversight.

\subsubsection{Summary}

In summary, NIST's work indicates that EUVL optics are susceptible to potential energy damage due to the neutralization of highly charged ions. Initial estimates for damage yields suggest that $\sim 20$ atoms may be removed for each $\mathrm{Xe}^{10+}$ ion, about an order of magnitude more than due to just kinetic energy alone. The removal of optical material results in an EUV reflectivity change of about $1 \%$ for $\sim 250 \mathrm{eV} / \mathrm{nm}^{2}$ deposited on the surface. This change is correlated with an increase in the surface conductivity measured by tunneling spectroscopy and appears to be a very sensitive measure for the change of the surface state that can be employed for studying a broader class of critical materials. Further efforts are expected to provide more quantitative guidance for mitigating potential energy damage effects.

\section{Acknowledgments}

The authors would like to acknowledge Dr. Chris Verzani's (NIST) assistance with EBIT ion beam operations and Andy Aquila's (LBNL) assistance with EUV reflectance mapping. Portions of this work were performed under the auspices of the US. Department of Energy by the University of California Lawrence Livermore National Laboratory under contract number W-7405-ENG-48. The work was partially funded by the International SEMATECH under the contract LITH 150 and LITH 160. 


\section{Biographies}

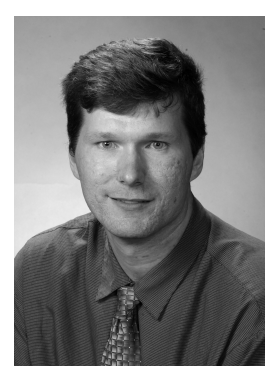

Joshua M. Pomeroy received his Ph.D. in physics from Cornell University in 2002. Dr. Pomeroy went to Los Alamos National Laboratory before joining the Plasma Radiation group at NIST (National Institute of Standards and Technology) in June of 2003. Dr. Pomeroy's research interests include fundamental physics of highly charged ions (HCIs), the interaction of HCIs with surfaces, and manipulation of surfaces by energetic processes during growth and erosion. Contact: joshua.pomeroy@nist.gov.

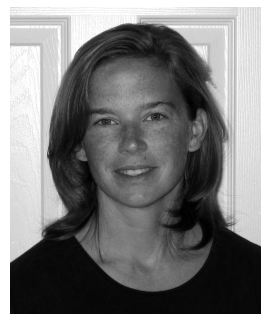

Laura P. Ratliff received her Ph. D. in physics from the University of Virginia in 1993. She then went to NIST to study laser cooling and trapping of neutral atoms. Her current research interests include the physics of highly charged ions (HCIs), the interactions of HCIs with surfaces and scanning tunneling microscopies. Contact:

laura.ratliff@nist.gov.

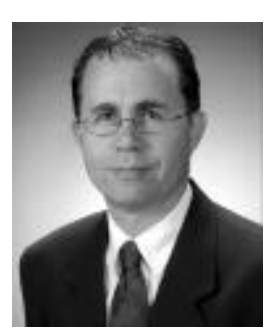

John D. Gillaspy received his Ph.D. from Harvard University in 1988. He came to the National Institute of Standards and Technology (NIST) as a National Research Council Postdoctoral Associate, before joining the NIST staff permanently in 1991 to lead the construction of the Electron Beam Ion Trap Facility. In 1999, he became the leader of the NIST Plasma Radiation Group (http://physics.nist.gov/gillaspy). Contact: john.gillaspy@nist.gov.

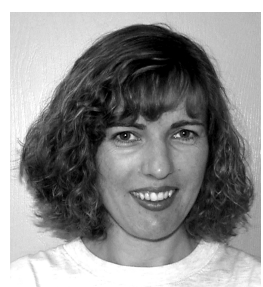

Saša Bajt received her Ph.D. in physics from the University of Heidelberg in Germany in 1990. She then worked for the University of Chicago at the National Synchrotron Light Source (NSLS) developing $x-$ ray fluorescence and micro $\mathrm{x}$-ray spectroscopy. Dr. Bajt was a recipient of a Hawley medal in 1999 for the innovation and application of microbeam XAFS to mineralogical research. She joined Lawrence Livermore National Laboratory in 1996 where she is leading a Multilayer Development Team. Her current research interests include development of multilayer coatings and capping layers for Extreme Ultraviolet Lithography (EUVL) and X-ray optics. Contact: bajt@llnl.gov. 
Figure 1: The $13.4 \mathrm{~nm}$ reflectivity of an EUVL mirror after exposure to a very low dose of highly charged ions ( 1 ion/250 $\mathrm{nm}^{2}$ ) shows evidence of damage. The yellow lobes at the bottom of the image correspond to the regions of $\mathrm{HCI}$ exposure. We believe this increase in the reflectivity is due to an ablation of the capping layer in those regions, which has removed some the oxide, effectively thinning the ruthenium capping layer.

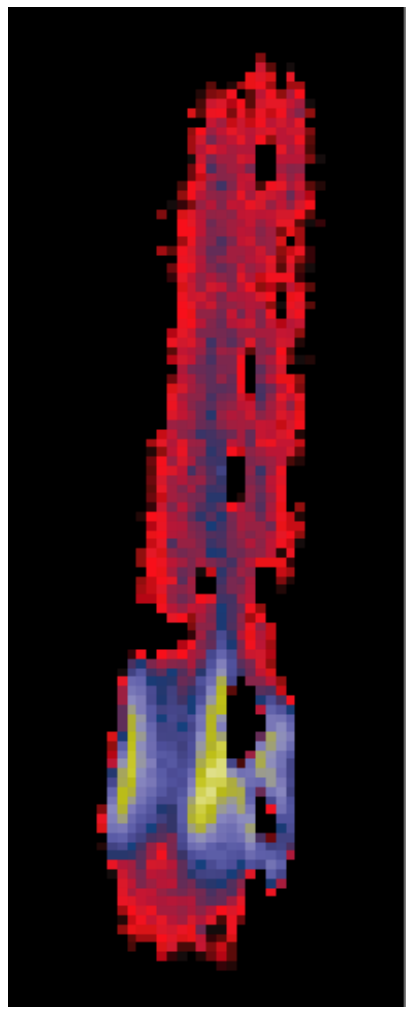

D $0.0 \%$

D $0.8 \%$ 

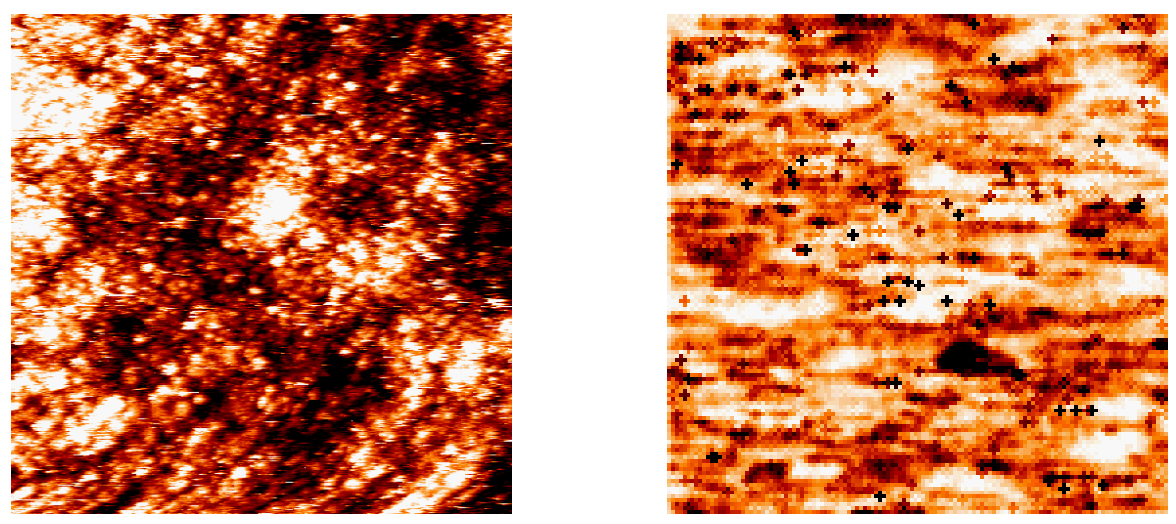

Figure 2: At left is a 200x200 nm STM image of an EUVL mirror after exposure to $\mathrm{Xe}^{10+}$ ions, the rich surface morphology and rapid kinetics mask morphological damage. At right is a spatial slice of spectroscopic data taken at $-0.63 \mathrm{~V}$ of bias: the image is a current map as a function of position, $20 \mathrm{~nm} \times 20$ $\mathrm{nm}$. The dark, high conductivity dots may be due to single ion impacts creating a low resistance pathway through the surface oxide. (The dots look like crosses due to nearest neighbor filtering used to reduce pixel noise.) 


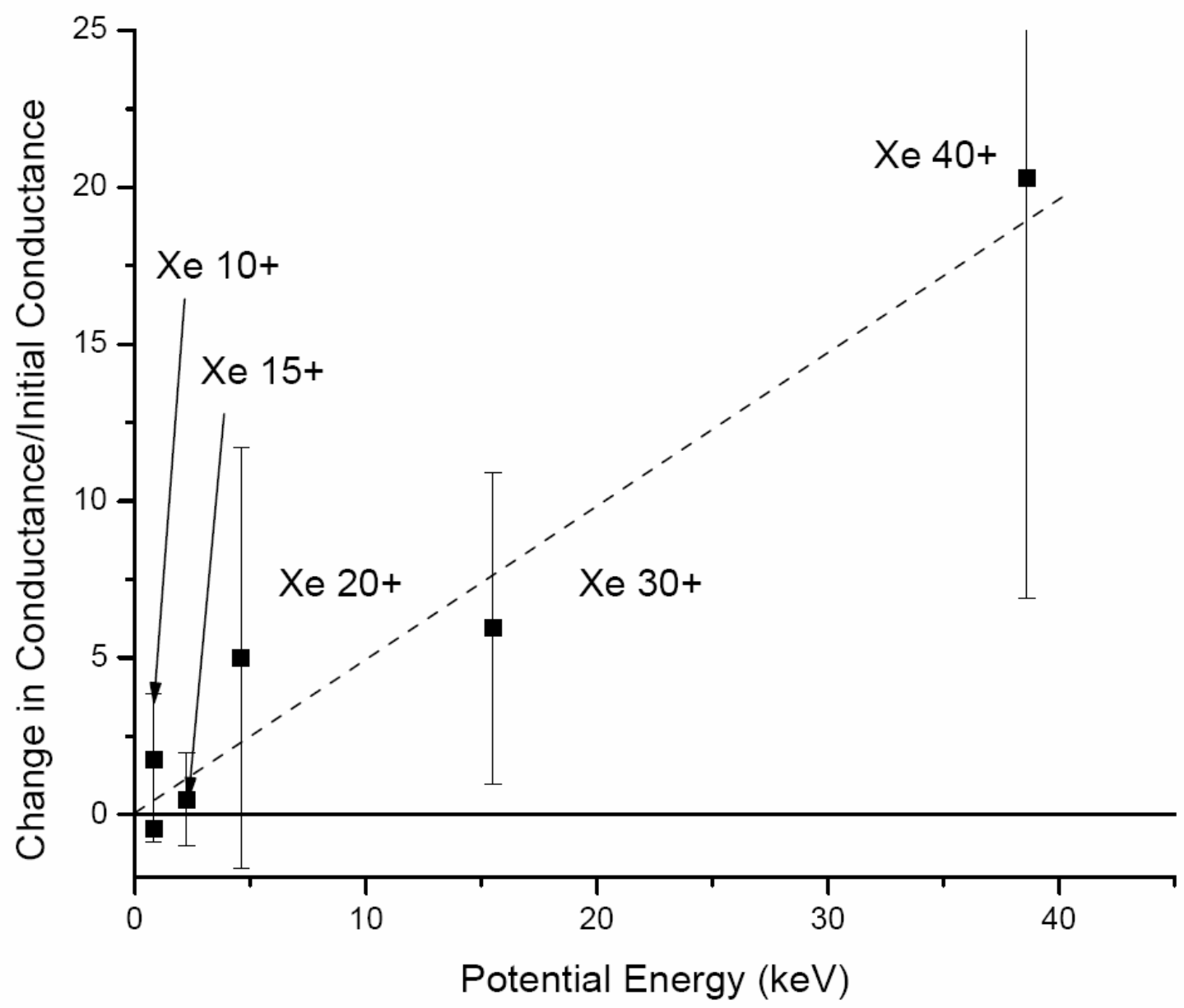

Figure 3: EUVL optics exposed to highly charged ions show an increase in the surface conductivity that increases systematically with potential energy density, i.e. the amount of neutralization energy deposited per unit surface area. The increasing surface conductivity is likely due to increased removal of the surface oxide. It is not believed this data suggests any kind of charge or energy threshold. Error bars represent the propagated standard deviations of the surface conductivity data, and are not uncertainties of the mean.

1 J.D. Gillaspy, "Highly Charged Ions," J. Phys. B: At. Mol. Opt. Phys. 34, R93-R130 (2001).

2 J. Pankert, et. al., "Status of Philips' extreme UV source," SPIE conference proceedings, 2004 in press.

${ }^{3}$ For example: N. Matsunami, et. al., "Energy Dependence of the Ion-Induced Sputtering Yields of Monatomic Solids," Atomic Data and Nuclear Data Tables 31, 1-80 (1984).

${ }^{4}$ P. Sigmund, "Theory of Sputtering. I. Sputtering Yield of Amorphous and Polycrystalline Targets," Phys. Rev. 184, 384-416 (1969). 
${ }^{5}$ J. Bohdansky, "A Universal Relation for the Sputtering Yield of Monatomic Solids ar Normal Incidence," Nucl. Instrum. and Meth. in Phys. Res. B2, 587-591 (1984).

${ }^{6}$ The Stopping and Range of Ions in Solids, J. F. Ziegler, J. P. Biersack and U. Littmark, Pergamon Press, New York, 1985; SRIM available at http://www.SRIM.org.

${ }^{7}$ W. Eckstein and R. Preuss, "New fit formulae for the sputtering yield," Journal of Nuclear Materials 320, 209-213 (2003).

${ }^{8}$ T. Schenkel, et. al., "Interaction of slow, very highly charged ions with surfaces," Prog. In Surf. Sci. 61, 23-84 (1999).

9 J. Burgdörfer, P. Lerner, and F. Meyer, "Above-surface neutralization of highly charged ions: The classical over-the-barrier model," Phys. Rev. A 44, 5674-5685 (1991).

${ }^{10}$ D.G.H. Schneider and M.A. Briere, "Investigations of the interactions of highest charge state ions with surfaces," Phys. Scr. 53, 228-242 (1995).

${ }^{11}$ L.P. Harris, Chapter 4, "Arc Cathode Phenomena," in VACUUM ARCS Theory and Applications, edited by J.M. Lafferty, John Wiley and Sons, New York, 120-168 (1980), e.g. p. 121.

${ }^{12}$ T. Schenkel, et al., "Emission of secondary particles from metals and insulators at impact of slow highly charged ions," Nucl. Instrum. \& Meth. B 125, 153-158 (1997).

${ }^{13}$ J.-P. Briand, et. al., "Observation of Hollow Atoms or Ions above Insulator and Metal Surfaces," Phys. Rev. Lett. 77, 1452-1455 (1996).

${ }^{14}$ I.S. Bitenskii, M.N. Murakhmetov, and E.S. Parilis, "Sputtering of nonmetals by intermediate-energy multiply charged ions through a Coulomb explosion," Sov. Phys. Tech. Phys. 24, 618-620 (1979).

${ }^{15}$ H.P Cheng and J.D. Gillaspy, "Nanoscale modification of silicon surfaces via Coulomb explosion," Phys. Rev. B 55, 2628-2636 (1997).

${ }^{16}$ F. Aumayr, J. Burgdorfer, P. Varga, \& H.P. Winter; “Sputtering of insulator surfaces by slow highly charged ions: Coulomb explosion or defect mediated desorption?" Comm. At. Mol. Phys. 34, 201-209 (1999).

${ }^{17}$ P. Stampfli, "Electronic excitation and structural stability of solids," Nucl. Instrum. \& Meth. B 107, 138-145 (1996).

${ }^{18}$ P. Stampfli and K.H. Bennemann, "Theory for the instability of the diamond structure of Si, Ge, and C induced by a dense electron-hole plasma," Phys. Rev. B 42, 7163-7173 (1990).

${ }^{19}$ T. Schenkel, et. al., "Ablation of GaAs by Intense, Ultrafast Electronic Excitation from Highly Charged Ions," Phys. Rev. Lett. 81, 2590-2593 (1998).

${ }^{20}$ T. Neidhart, et. al., "Potential Sputtering of Lithium Fluoride by Slow Multicharged Ions," Phys. Rev. Lett. 74, 5280-5283 (1995).

${ }^{21}$ M. Sporn, et. al., "Potential Sputtering of Clean $\mathrm{SiO}_{2}$ by Slow Highly Charged Ions," Phys. Rev. Lett. 79, 945-948 (1997).

${ }^{22}$ R.T. Williams, K.S. Song, W.L. Faust, and C.H. Leung, "Off-center self-trapped excitons and creation of lattice defects in alkali halide crystals," Phys. Rev. B. 33, 7232-7240 (1986).

${ }^{23}$ F. Aumayr and H. Winter, "Potential Sputtering," Phil. Trans. R. Soc. Lond., 362, 77-102 (2004).

${ }^{24}$ G. Hayderer, et. al., "Kinetically Assisted Potential Sputtering of Insulators by Highly Charged Ions," Phys. Rev. Lett. 86, 3530-3533 (2001).

${ }^{25}$ N. Itoh, "Bond scission induced by electronic excitation in solids: A tool for nanomanipulation," Nucl. Instrum \& Meth. B 122, 405-409 (1998).

${ }^{26}$ T. Schenkel, et. al., "Synergy of Electronic Excitations and Elastic Collision Spikes in Sputtering of Heavy Metal Oxides," Phys. Rev. Lett. 80, 4325-4328 (1998).

${ }^{27}$ R. Minniti, L.P. Ratliff, and J.D. Gillaspy, "In-situ Observation of Surface Modification induced by Highly Charged Ion Bombardment," Physica Scripta T92, 22-26 (2001).

28 A.I. Pikin, et. al,;"A beam line for highly charged ions," Rev. Sci. Instrum. 67, 2528-2533 (1996).

${ }^{29}$ L.P. Ratliff, et. al., "Continuous highly-charged ion beams from the NIST EBIT," Rev. Sci. Instrum. 68, 1998-2002 (1997).

${ }^{30} \mathrm{~S}$. Bajt, et. al., "Design and performance of capping layers for extreme-ultraviolet multilayer mirrors," Appl. Opt. 42, 5750-5758 (2003). 
${ }^{31}$ T. Schenkel, Chapter 8 "Surface Analysis with Slow Highly Charged Ions like $\mathrm{Au}^{69+}$ : TOF-SIMS and the Probing of Nano-Environments," Ion Beam Analysis of Surfaces and Interfaces of Condensed Matter Systems edited by P. Chakraborty, 34-82 (2003) and references therein. 